\title{
Inflammatory fibroid polyp of the duodenum: is endoscopic resection a feasible therapeutic choice?
}

\author{
Faisal Inayat, ${ }^{1}$ Maham Ashraf Khan, ${ }^{1}$ Fahad Zafar, ${ }^{2}$ Ahmed Munir ${ }^{3}$
}

'Allama Iqbal Medical College, Lahore, Pakistan

${ }^{2}$ King Edward Medical University, Lahore, Pakistan ${ }^{3}$ Services Institute of Medical Sciences, Lahore, Pakistan

\section{Correspondence to Dr Faisal Inayat, faisalinayat@hotmail.com}

This case was presented as an abstract at the Annual Scientific Meeting of the American College of Gastroenterology, 13-18 October 2017, in Orlando, FL, USA

Accepted 31 October 2018

\section{DESCRIPTION}

A 52-year-old man was referred to our medical centre for the evaluation of persistent dyspepsia for several months. He denied nausea, vomiting, regurgitation, weight loss, haematemesis or melena. He had no history of pre-existing gastrointestinal disorders or non-steroidal anti-inflammatory drug use. His family history was negative for gastrointestinal cancer. In his prior work-up, stool antigen testing was negative for Helicobacter pylori infection. Despite a 6-week trial of proton-pump inhibitor therapy, his symptoms persisted. At presentation, the clinical examination was unremarkable. Laboratory studies for haematology, serum biochemistry and urine analysis were within normal limits.

Esophagogastroduodenoscopy (GIF-H190; Olympus Medical Systems, Center Valley, Pennsylvania, USA) showed a large 6-cm submucosal pedunculated polyp with a broad base, located in the duodenal bulb area with the stalk attached just distal to the pylorus (figure 1). Endoscopic ultrasonography (EUS) showed a hypoechoic, homogeneous lesion, with indistinct margins arising from the second and third echolayers of the duodenal wall (figure 2). Fine-needle aspiration was inconclusive for the exact aetiology. Due to the pedunculated form, a decision was made for endoscopic removal of the lesion. Therein, an uneventful endoscopic submucosal dissection was performed followed by endoloop placement. No immediate complications of the procedure were noted.

Pathological examination showed the polypoid mass expanding the duodenal submucosa (figure 3). The proliferation of mononuclear, spindle-shaped cells formed a characteristic 'onion skin' arrangement around blood vessels (figure 4).

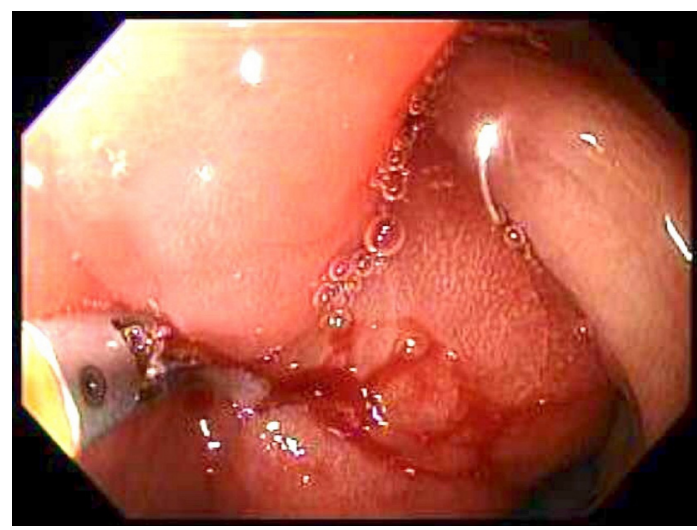

Figure 1 Oesophagogastroduodenoscopy showing a 6-cm submucosal pedunculated polyp with a broad base, located in the duodenal bulb area.

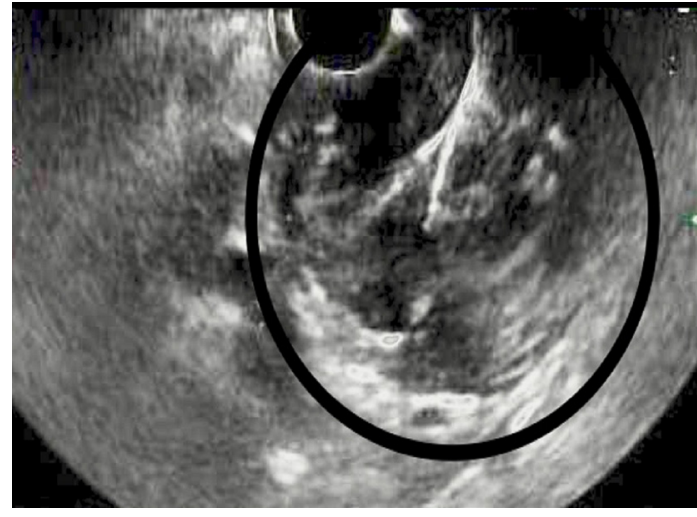

Figure 2 Endoscopic ultrasound showing a homogeneous hypoechoic lesion, with indistinct margins arising from the second and third sonographic layers of the duodenal wall.

The inflammatory infiltrate was predominantly composed of eosinophils (figure 5). On immunohistochemistry, the spindle cells were diffusely positive for vimentin and CD34, and negative for CD117, SMA, S100, AE1/AE3 and factor VIII. These pathological findings were consistent with the diagnosis of inflammatory fibroid polyp (IFP). The patient recovered well and he was discharged from the hospital after 24 hours. At the 2-week follow-up, he reported significant improvement in dyspepsia symptoms.

IFP is a rare benign mesenchymal lesion of the gastrointestinal tract. Although it was initially reported by Vanek in 1949, Helwig and Ranier first coined the term inflammatory fibroid polyp in 1953. Since then, IFP has gained acceptance in the medical literature. These lesions can be found in any

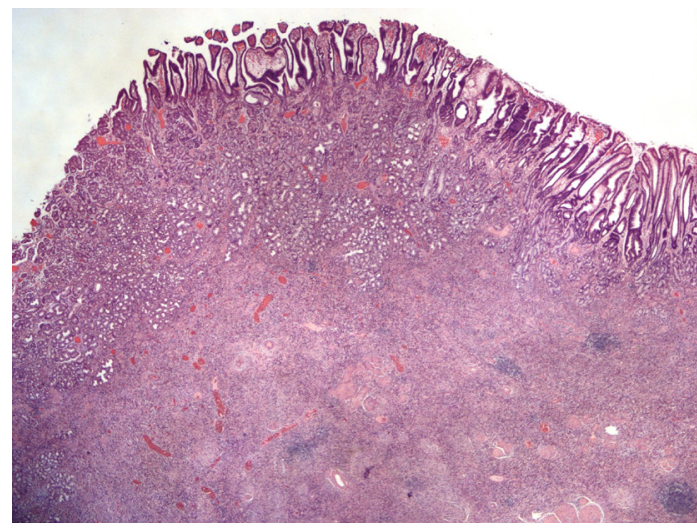

Figure 3 Pathological examination of the resected duodenal specimen showing the polypoid mass expanding the duodenal submucosa $(\mathrm{H} \& \mathrm{E} ; \times 40)$. 


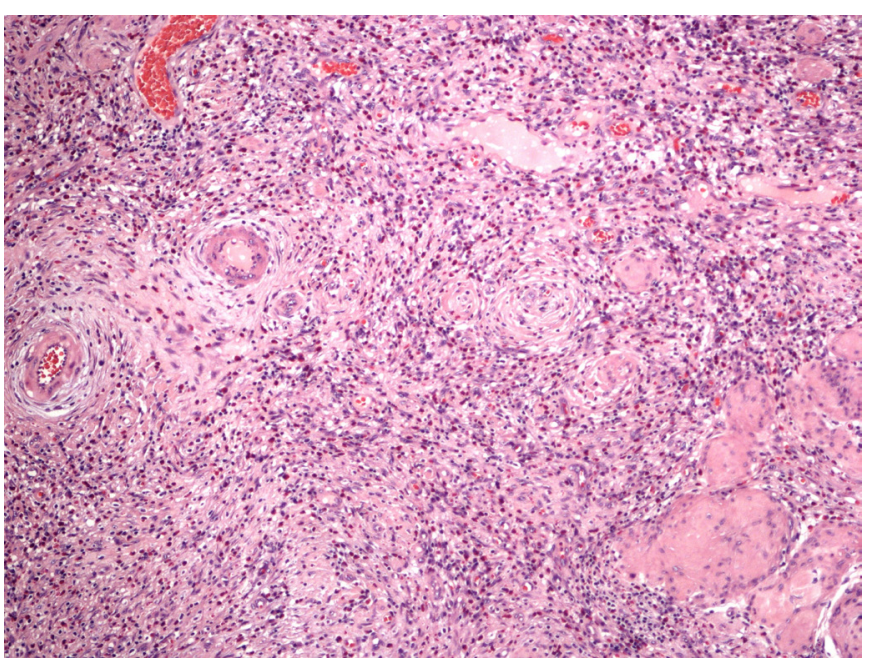

Figure 4 Mononuclear, spindle-shaped cells with an 'onion skin' appearance around blood vessels ( $\mathrm{H} \& \mathrm{E} ; \times 200)$.

age group, but peak incidence is between the sixth and seventh decades of life, with a slight male predominance. IFPs may occur in all parts of the gastrointestinal tract. The most common location is the stomach $(66 \%-75 \%)$, followed by the small intestine, predominantly ileum (18\%-23\%), colon and rectum (4\%-7\%), gallbladder (1\%), oesophagus (1\%) and appendix. ${ }^{1}$

IFP of the duodenum is a remarkably rare occurrence. To the best of our knowledge, only eight adult cases of duodenal IFPs have been reported as of June 2018. ${ }^{1}$ The exact pathogenesis of this disease remains unclear. Although the clinical presentation varies by location and size, a vast majority of the lesions are typically asymptomatic and are incidentally discovered by endoscopy or during a laparotomy, in patients hospitalised with other medical conditions. Duodenal IFPs frequently present with non-specific features of abdominal pain, dyspepsia and/or upper gastrointestinal blood loss. ${ }^{1}$

Endoscopy typically reveals a solitary polypoid or sessile, intramural lesion, with a smooth or eroded mucosa. ${ }^{1}$ EUS has emerged as a promising diagnostic tool, especially for gastric IFPs. On EUS, these lesions are hypoechoic and homogeneous with indistinct margins, and are usually located within the second and third echolayers of the mucosal wall. ${ }^{1}$ Furthermore, internal

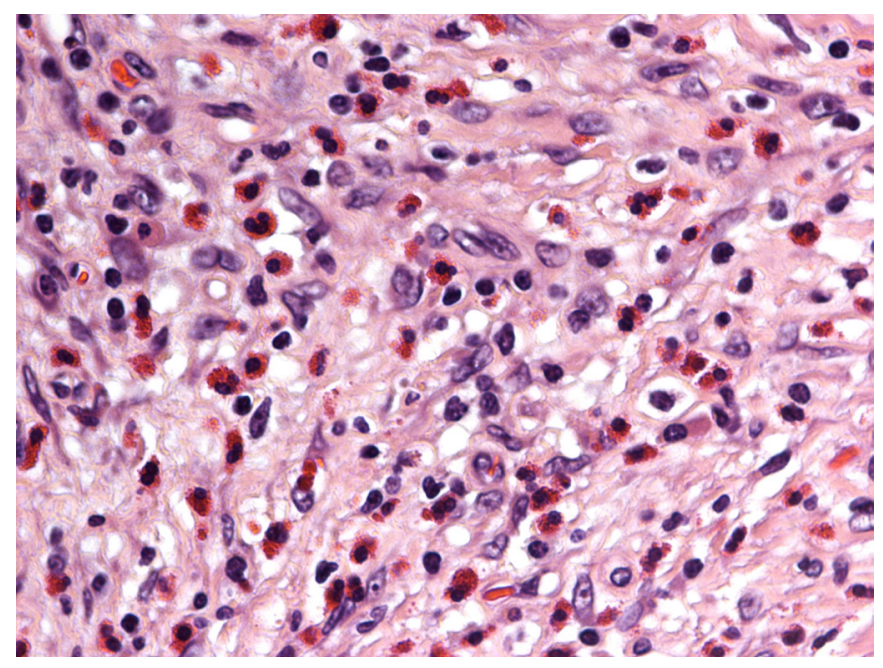

Figure 5 Inflammatory infiltrate was predominantly composed of eosinophils $(H \& E ; \times 400)$. echoes may also be present, which correspond to the numerous blood vessels within the lesion. Gastrointestinal stromal tumour (GIST) and lipomas are among the major differentials. On EUS, the GISTs are transmural and lipomas have well-defined margins. ${ }^{1}$ Therefore, an expert endoscopist may use EUS for initial detection of gastrointestinal IFPs that may help in diagnosing these lesions preoperatively. However, histopathological analysis is usually required to confirm the diagnosis. It is further notable that biopsies can be challenging as the epicentre of the lesion is mostly in the submucosa and the IFP polyp is often covered by epithelial mucosa. On immunohistochemistry, spindle cells of IFPs are generally positive for CD34 and negative for S-100 protein, P53, CD117 and Bcl-2.

In regards to the management, complete resection is necessitated due to the rapid growth observed in these lesions that may lead to intestinal obstruction. ${ }^{2}$ Endoscopic polypectomy is the preferred technique in polypoid lesions of up to $2 \mathrm{~cm}$, usually arising from the stomach or colon. However, surgical resection may be considered in patients with large, sessile, submucosal polyps as the risk of incomplete resection and gastrointestinal perforation is relatively higher following endoscopic resection in this group. ${ }^{2}$ Extended follow-up is required to further evaluate prognosis of this disease.

The data on successful endoscopic resections are scarce, comprising a handful of case reports. Nakase et al undertook an uneventful endoscopic resection of a caecal IFP lesion that measured $4.5 \mathrm{~cm} .^{3}$ Similarly, Kayyali et al reported an interesting case of a giant colonic IFP (size: $7.0 \times 4.0 \times 3.0 \mathrm{~cm}$ ) that was successfully removed endoscopically in a piecemeal fashion and the lesion site was tattooed, without complications or recurrence. ${ }^{4}$ Owing to the benign clinical behaviour of IFPs and the low post-endoscopic resection recurrence rate, endoscopic polypectomy could be an appropriate approach in these patients if technically possible, considering the size, location and the morphology of the polyp. In addition to relieve symptoms, endoscopic polypectomy may also be used as a valuable diagnostic technique for providing tissue specimens for accurate histological examination to resolve diagnostic uncertainty. Moreover, it is also a feasible alternative for patients who are not surgical candidates or refuse surgery.

In the present case, EUS was very suggestive of the diagnosis of IFP before removal, based on the consistent endoscopic and EUS features. Although the size of the polyp was considerably large $(6 \mathrm{~cm})$, pedunculated morphology made it amenable for endoscopic resection. Thus, the lesion was successfully removed endoscopically, avoiding major bowel surgery as that is associated

\section{Learning points}

- Duodenal IFP is a rare condition, which may mimic malignant spindle-cell tumours on superficial biopsies. To our research, the present report represents the ninth documented case of IFP of the duodenum in the published medical literature.

- Although it is a benign clinical entity, clinicians and surgeons should be vigilant for this disease and it should be included in the differential diagnoses of all duodenal masses. EUS may improve the ability to make a preoperative diagnosis of IFP.

- A timely and correct aetiology establishment may enable the endoscopist to undertake endoscopic resection, using a case-by-case approach, as it can avoid unnecessary surgical intervention in selected patients. Further research is warranted to validate these findings. 
with relatively higher morbidity and mortality. As highlighted in this report, endoscopic resection may be possible in patients with relatively larger duodenal IFP lesions, but the expertise of an endoscopist plays a key role in determining the clinical success of the procedure.

Contributors Fl: designed the study, performed the literature review, drafted the manuscript, revised the manuscript critically for important intellectual content and gave the final approval for the version published. MAK: performed the literature review and drafted the manuscript. FZ: contributed to and reviewed the manuscript. AM: reviewed the manuscript.

Funding The authors have not declared a specific grant for this research from any funding agency in the public, commercial or not-for-profit sectors.
Competing interests None declared.

Patient consent Obtained.

Provenance and peer review Not commissioned; externally peer reviewed.

\section{REFERENCES}

1 Wysocki AP, Taylor G, Windsor JA. Inflammatory fibroid polyps of the duodenum: a review of the literature. Dig Surg 2007:24:162-8.

2 Zinkiewicz K, Zgodzinski W, Dabrowski A, et al. Recurrent inflammatory fibroid polyp of cardia: a case report. World I Gastroenterol 2004;10:767-8.

3 Nakase H, Mimura J, Kawasaki T, et al. Endoscopic resection of small inflammatory fibroid polyp of the colon. Intern Med 2000;39:25-7.

4 Kayyali A, Toumeh A, Ahmad U, et al. Giant inflammatory fibroid polyp of the descending colon treated with endoscopic resection. ACG Case Rep J 2013;1:36-9.

Copyright 2018 BMJ Publishing Group. All rights reserved. For permission to reuse any of this content visit https://www.bmj.com/company/products-services/rights-and-licensing/permissions/

BMJ Case Report Fellows may re-use this article for personal use and teaching without any further permission.

Become a Fellow of BMJ Case Reports today and you can:

- Submit as many cases as you like

- Enjoy fast sympathetic peer review and rapid publication of accepted articles

- Access all the published articles

- Re-use any of the published material for personal use and teaching without further permission

For information on Institutional Fellowships contact consortiasales@bmjgroup.com

Visit casereports.bmj.com for more articles like this and to become a Fellow 